\title{
VEGF/VEGFR2 blockade does not cause retinal atrophy in AMD-relevant models
}

\author{
Da Long, Yogita Kanan, Jikui Shen, Sean F. Hackett, Yuanyuan Liu, Zibran Hafiz, Mahmood Khan, \\ Lili Lu, and Peter A. Campochiaro
}

Department of Ophthalmology, and Department of Neuroscience, Johns Hopkins University School of Medicine, Baltimore, Maryland, USA.

\begin{abstract}
Intraocular injections of VEGF-neutralizing proteins provide tremendous benefits in patients with choroidal neovascularization (NV) due to age-related macular degeneration (AMD), but during treatment some patients develop retinal atrophy. Suggesting that VEGF is a survival factor for retinal neurons, a clinical trial group attributed retinal atrophy to VEGF suppression and cautioned against frequent anti-VEGF injections. This recommendation may contribute to poor outcomes in clinical practice from insufficient treatment. Patients with type 3 choroidal NV have particularly high risk of retinal atrophy, an unexplained observation. Herein we show in mouse models that VEGF signaling does not contribute to photoreceptor survival and functioning: (a) neutralization of VEGFR2 strongly suppresses choroidal NV without compromising photoreceptor function or survival; (b) VEGF does not slow loss of photoreceptor function or death in mice with inherited retinal degeneration, and there is no exacerbation by VEGF suppression; and (c) mice with type 3 choroidal NV develop retinal atrophy due to oxidative damage with no contribution from VEGF suppression. Intraocular injections of VEGF-neutralizing proteins, a highly effective treatment in patients with neovascular AMD, should not be withheld or reduced due to concern that they may contribute to long-term visual loss from retinal atrophy.
\end{abstract}

Authorship note: DL and YK contributed equally to this work.

Conflict of interest: $\mathrm{PAC}$ is a consultant to Aerpio Therapeutics, Alimera, Allegro, Allergan, Applied Genetic Technologies, AsclipiX, Astellas, Exonate, Genentech/ Roche, Intrexon, Merck, Novartis, and Rxi. PAC receives research funding from Aerpio Therapeutics, Alimera, Aerpio Therapeutics, Allergan, AsclipiX, Clearside, Genentech/Roche, Genzyme, Graybug, Oxford BioMedica, Regeneron, Regenxbio, and Rxi. PAC has equity in Allegro and Graybug. All of these potential conflicts of interests are managed by Johns Hopkins University.

Submitted: January 29, 2018

Accepted: April 12, 2018

Published: May 17, 2018

\section{Reference information:}

JCI Insight. 2018;3(10):e120231.

https://doi.org/10.1172/jci.

insight.120231.

\section{Introduction}

Age-related macular degeneration (AMD) is the most prevalent cause of moderate and severe vision loss in individuals older than 60 in developed countries (1). It is a neurodegenerative disease in which there is accumulation of extracellular material beneath retinal pigmented epithelial (RPE) cells followed by gradual death of photoreceptors and RPE cells. The extracellular material occurs diffusely resulting in thickening of Bruch's membrane, which separates the RPE and choroid, and focally as mounds called drusen that irregularly elevate RPE cells and photoreceptors and appear as yellow or white spots mostly in the macula but sometimes extending into mid-peripheral retina. There is gradual death of photoreceptors in the macula, with rod photoreceptor cell death greater than that of cone photoreceptors in the early stages (2). A subgroup of AMD patients show dramatic signs of cell death manifested by visible patches that appear lighter than surrounding retina where there is regional loss of photoreceptors, RPE cells, and choriocapillaris, a process referred to as geographic atrophy (GA; for review see ref. 3). It usually starts as a single or multiple atrophic patches in an extrafoveal location that gradually enlarge and when multiple, often coalesce to form a partial ring that over time extends into the fovea causing reduction in visual acuity.

Another subgroup of AMD patients that overlaps with the GA subgroup develops choroidal neovascularization (NV), and is classified as having neovascular AMD (NVAMD). Type 1 choroidal NV grows from the choroid through Bruch's membrane under the RPE. Type 2 choroidal NV grows from the choroid through Bruch's membrane and the RPE into the subretinal space. Type 3 choroidal NV, also called retinal angiomatous proliferation (RAP), grows in the opposite direction from the deep capillary bed of retinal vessels, through the outer nuclear layer (ONL) that contains the cell bodies of the photoreceptors into the subretinal space (4). Choroidal NV leaks fluid into and under the retina causing reduced vision that is initially reversible, but if left untreated the NV tends to grow and recruit RPE, glia, and fibroblasts resulting in a fibrovascular scar that damages photoreceptors resulting in severe, irreversible loss of central vision.

Vascular endothelial growth factor (VEGF) is an important stimulus in mouse and primate models of type 2 choroidal NV $(5,6)$ and increased expression of VEGF in photoreceptors results in type 3 choroidal 
NV (7). Intraocular injection of VEGF-neutralizing proteins reduces leakage from choroidal NV and markedly improves visual acuity, but as intraocular levels drop, leakage usually returns necessitating repeated injections $(8,9)$. In initial clinical trials, visual benefits were maintained during 2 years of monthly injections of ranibizumab, but during an extension study in which injections could be given as infrequently as every 3 months, all visual gains were lost (10). Despite the outstanding benefit from VEGF suppression and evidence that reducing the frequency of injections too much was harmful, there was concern that suppression of VEGF in the retina was dangerous, because a study in mice suggested that systemic injection of an adenoviral vector expressing soluble VEGFR1 caused photoreceptor degeneration (11). Also, conditional knockout of VEGF in RPE cells results in rapid loss of choriocapillaris and retinal degeneration (12). In contrast, transgenic mice in which the rhodopsin promoter was used to express high levels of a potent VEGF-neutralizing protein in the retina for up to 7 months, showed no effects on the choriocapillaris and no loss of photoreceptor cell function nor photoreceptor cell death (13). Also, sustained suppression of VEGFR signaling by repeated administration of a VEGFR tyrosine kinase inhibitor caused no reduction in photoreceptor function or survival (14). Despite the conflicting evidence in the literature, clinicians seemed to be concerned about toxicity as well as cost and sought ways to reduce the frequency of injections. One approach was to have patients return at monthly intervals, but only inject a VEGF-neutralizing protein when optical coherence tomography (OCT) demonstrated intraretinal or subretinal fluid in the macula. This treatment regimen was named pro re nata $(\mathrm{PRN})$. A large multicenter clinical trial, the Comparison of Age-related Macular Degeneration Treatments Trials (CATT), compared 4 treatment arms in patients with NVAMD, monthly ranibizumab, monthly bevacizumab, PRN ranibizumab, and PRN bevacizumab. After 2 years, there was no difference between ranibizumab and bevacizumab when the dosing regimen was the same, but visual outcomes were significantly better in subjects treated with monthly injections of either agent compared with PRN injections of the same agent (15). It was also noted that many patients had new onset of depigmented spots in the macula identified by fundus photography and fluorescein angiography and the percentage of subjects in which they occurred was greater in the monthly injection groups than the PRN groups. The authors referred to these spots as GA, which implies photoreceptor and RPE cell death in the region of the depigmented spots, but no evidence of loss of these cells was provided. The authors concluded, "The development of geographic atrophy was higher in both monthly treated groups than in the as-needed groups. This may be important to consider when weighing the risks and benefits of monthly versus as-needed treatment and in the selection of drug." Usually when there is a difference in visual outcome among treatment groups, it is recommended that the regimen providing the best outcome be used in clinical practice, but in this unprecedented move, the CATT research group seemed to be recommending against monthly injections because of a higher risk of GA even though visual outcomes were better in the monthly injection groups than in the PRN groups. In a follow-up study, it was found that 1 of 5 patients enrolled in CATT developed hypopigmented spots labeled as GA during the 2 year treatment period and increased risk of their development was associated with poor visual acuity at baseline, type 3 choroidal NV, foveal intraretinal fluid, monthly dosing, and treatment with ranibizumab (16). The CATT study group stated, "Because VEGF plays an important role in the normal function of the retina and the maintenance of the choriocapillaris by the RPE, therapies that block VEGF could have an effect on the development and progression of GA," indicating that despite conflicting evidence as to whether VEGF is a survival factor for photoreceptors, they considered the issue settled. They concluded, "These findings have important clinical implications and should be included in discussions with patients regarding the benefits and risks of the choice of treatment type and regimen. Although monthly injections may result in slightly better visual outcomes at 2 years, the increased risk of GA development may offset this benefit long term."

Thus, the hypothesis that VEGF is a survival factor for photoreceptors, on which the recommendation against frequent anti-VEGF injections was based, is of major clinical importance. There are several proteins that have been demonstrated to promote photoreceptor survival including fibroblast growth factor 2 (FGF2), ciliary neurotrophic factor (CNTF), glial cell line-derived neurotrophic factor (GDNF), and most recently rod-derived cone viability factor (rdCVF). The following types of evidence have led to these conclusions. (a) Blockade of the receptor for the protein on photoreceptors causes loss of function and/or death. This was demonstrated for FGF2 (17). (b) Injection of the protein into the vitreous or increasing expression of the protein in the eyes of mice or rats with retinal degeneration slows the rate of photoreceptor loss of function and death. This has been demonstrated for FGF2, CNTF, GDNF, and rdCVF (18-24). In this study, we sought to use these previously established criteria to determine if VEGF is a survival factor for photoreceptors. 


\section{Results}

Inhibition of VEGFR2 suppresses choroidal NV without photoreceptor damage. It has been hypothesized that activation of VEGFR2 on photoreceptors promotes photoreceptor survival and that neutralization of VEGF or VEGFR2 may damage photoreceptors by reducing VEGFR2-derived survival signals (11). To test this hypothesis, 3 intraocular injections a week apart of $40 \mu \mathrm{g}$ of an antibody directed against human VEGFR2 that cross-reacts with rat VEGFR2 was given in one eye and $40 \mu \mathrm{g}$ of control IgG was injected in the fellow eye of Brown Norway rats. One week after the third injection of anti-VEGFR2, fundus photographs and OCT scans were unchanged from baseline and appeared normal (Figure 1A). In particular, postinjection fundus photographs showed no hypo- or hyperpigmented spots typical of retinal atrophy or photoreceptor degeneration and OCT scans showed no thinning of the retina characteristic of retinal atrophy. Electroretinograms (ERGs) at the same time point showed no significant differences between IgG-injected and antiVEGFR2-injected eyes in mean scotopic a-wave amplitudes (Figure 1B), indicating no difference in rod photoreceptor function; mean scotopic b-wave amplitudes (Figure 1C), indicating no difference in secondorder neurons in the rod pathway; or photopic b-wave amplitudes (Figure 1D), indicating no difference in cone photoreceptor function. Rats were then euthanized and ONL thickness was measured at 6 locations along the vertical meridian through the optic nerve. There was no significant difference in mean ONL thickness at each of the 6 locations (Figure 1E), indicating that suppression of VEGFR2 for 4 weeks did not cause any photoreceptor cell death. Another cohort of Brown Norway rats had laser-induced rupture of Bruch's membrane at 4 locations in each eye followed by intravitreous injection of $20 \mu \mathrm{g}$ of anti-VEGFR2 in one eye and $20 \mu \mathrm{g}$ of control IgG in the fellow eye, or $40 \mu \mathrm{g}$ of aflibercept in one eye and PBS in the fellow eye. Two weeks after injection there were small areas of choroidal NV at Bruch's membrane rupture sites in eyes injected with anti-VEGFR2 and large areas of choroidal NV in eyes injected with IgG or PBS (Figure $1 \mathrm{~F}$ ). The mean area of choroidal NV was significantly reduced in eyes injected with anti-VEGFR2 compared with fellow eyes injected with IgG, and significantly less in eyes injected with aflibercept compared with fellow eyes injected with PBS (Figure 1G).

Effect of VEGF or VEGFR2 suppression on photoreceptor function or survival in mice with inherited retinal degeneration. While the above indicates that suppression of VEGFR2 does not reduce photoreceptor function or survival in normal retina, it does not rule out a role for VEGFR signaling in the survival of photoreceptors that are under stress. Rd10 mice have a loss-of-function mutation in Pde6b (cGMP phosphodiesterase 6B, rod receptor, $\beta$ polypeptide) that causes rod photoreceptor cell death with most eliminated by P35 $(25,26)$. Mutations in $P d e 6 b$ also cause retinitis pigmentosa in humans (27). Intravitreous injection of agents that promote survival of photoreceptors delay cell death in models of inherited retinal degeneration $(18,19)$. To determine if high levels of VEGF provide any photoreceptor protection in $r d 10$ mice, they were given intravitreous injections of 100 ng of recombinant VEGF $\left(\mathrm{VEGF}_{165}\right)$ in one eye and PBS in the fellow eye at P14, P21, and P28. At P35, there were no significant differences in mean scotopic a-wave (Figure 2A) or b-wave amplitude (Figure 2B), or mean photopic b-wave amplitude (Figure 2C), indicating that high levels of VEGF do not protect against loss of photoreceptor function in $r d 10$ mice. A related question is whether suppression of VEGFR signaling would hasten loss of photoreceptor function in $r d 10$ mice. Rd10 mice were given subcutaneous injections of $5 \mu \mathrm{g}$ of SU4312, a VEGFR tyrosine kinase inhibitor, every 5 days starting at P14. This dosing regimen was selected because it was previously demonstrated to strongly suppress ocular NV (14). At P35, there were no significant differences in mean scotopic a-wave (Figure 2D) or b-wave amplitude (Figure 2E), or mean photopic b-wave amplitude (Figure $2 \mathrm{~F}$ ), indicating that suppression of VEGF signaling does not exacerbate loss of photoreceptor function in $r d 10$ mice. Rhodopsin kinase (GRK1) plays an important role in phototransduction and its level in the retina provides an assessment of rod photoreceptor cell survival and function. Rd10 mice were given intravitreous injections of $100 \mathrm{ng}$ of recombinant $\mathrm{VEGF}_{165}$ in one eye and vehicle in the fellow eye at P14, P21, and P28, or they were given subcutaneous injections of $5 \mu \mathrm{g}$ of SU4312, a VEGFR tyrosine kinase inhibitor, every 5 days starting at P14. At P35, GSK1 immunoblots of retinal homogenates showed similar levels of GSK1 in retinas of $r d 10$ mice that had been treated with high levels of VEGF, vehicle, or a VEGFR tyrosine kinase inhibitor (Figure 2G). Densitometry confirmed that treatment of $r d 10$ mice with high levels of VEGF or suppression of VEGF signaling had no effect on rod survival or function (Figure $2 \mathrm{H}$ ).

Type 3 choroidal NV causes oxidative damage and photoreceptor cell death. Type 3 choroidal NV was found to be a risk factor for the development of GA in the CATT study (16). This observation sheds doubt on the hypothesis that suppression of VEGF is the cause of retinal atrophy in patients with NVAMD, because why would a particular type of choroidal NV be more susceptible to VEGF-induced damage? It is worthwhile to 
A
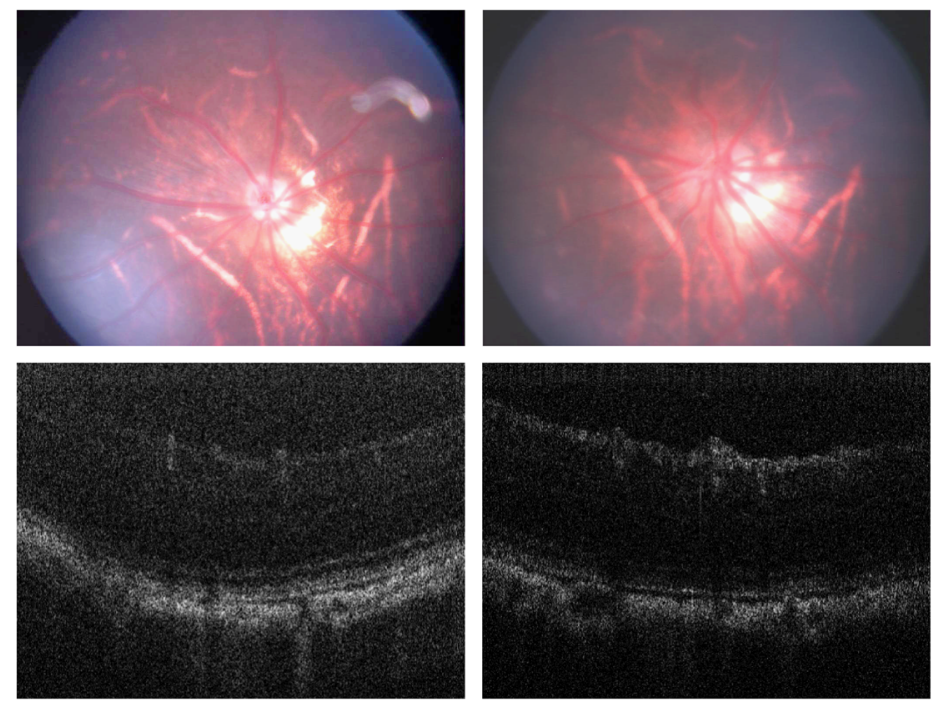

B
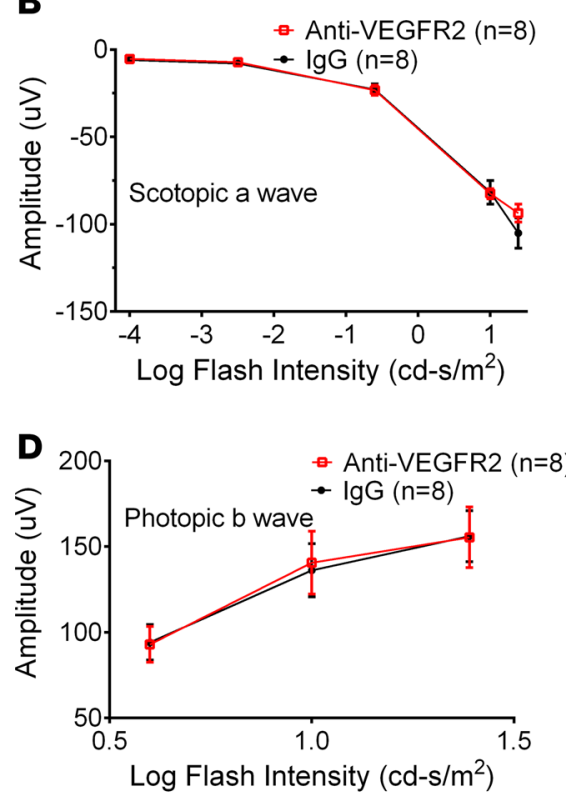

F
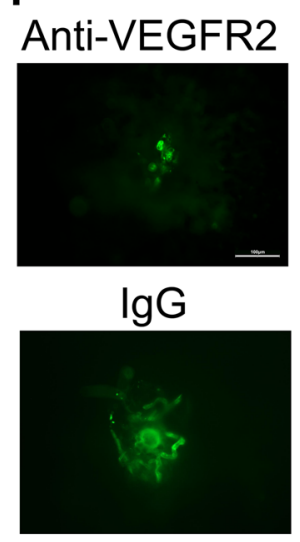

After 3 Injections

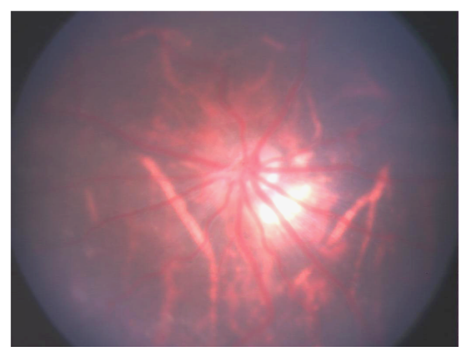

C

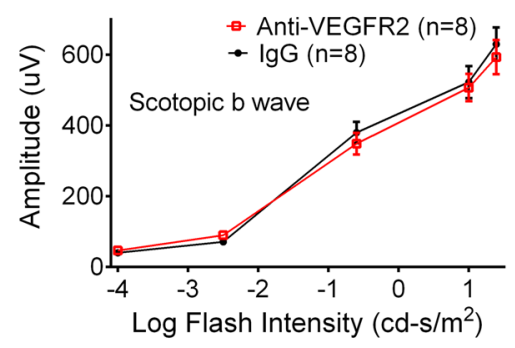

E

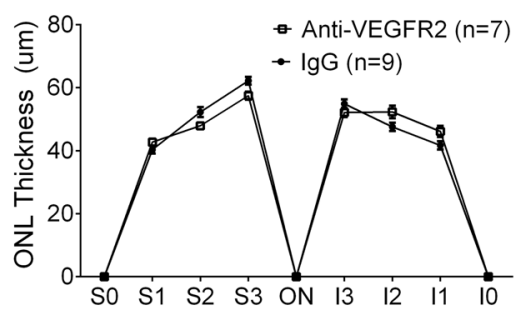

G

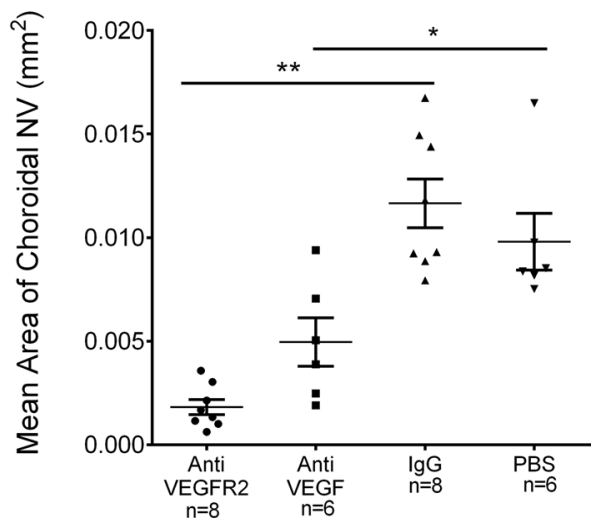

Figure 1. Inhibition of VEGFR2 suppresses choroidal neovascularization (NV) without photoreceptor damage. Brown Norway rats had fundus photography (A, top left) and optical coherence tomography (A, bottom left) through the posterior pole at baseline and then were given 3 intravitreous injections a week apart of $40 \mu \mathrm{g}$ of a neutralizing antibody directed against VEGFR2 in one eye and control IgG in the fellow eye. One week after the third injection, repeat fundus photography (A, top right) and optical coherence tomography (A, bottom right) showed no change in appearance or thickness of the retina. Electroretinography showed that compared with control IgG-injected eyes, those injected with anti-VEGFR2 had no significant differences in mean scotopic a-wave amplitudes (B), mean scotopic b-wave amplitudes (C), or mean photopic b-wave amplitudes (D) at any of several flash intensities tested. Rats were euthanized and outer nuclear layer (ONL) thickness was measured at 6 locations in the 12:00 to $6: 00$ meridian passing through the optic nerve. There were no significant differences in mean ONL thickness at any of the 6 locations in anti-VEGFR2-injected eyes versus those injected with control IgG (E). Brown Norway rats had laser-induced rupture of Bruch's membrane at 4 locations in one eye followed by an intraocular injection of $20 \mu \mathrm{g}$ anti-VEGFR2 $(n=8), 20 \mu \mathrm{g}$ of control IgG $(n=8), 40 \mu \mathrm{g}$ of aflibercept (anti-VEGF, $n=6)$, or PBS $(n=6)$. After 14 days, rats were euthanized and choroidal flat-mounts were stained with FITC-labeled Griffonia simplicifolia agglutinin lectin. Representative images show small areas of choroidal NV in eyes that had been injected with antiVEGFR2 or aflibercept (anti-VEGF) and large areas of choroidal NV in eyes that had been injected with control IgG or PBS (F). Image analysis showed that compared with eyes injected with control IgG, those injected with anti-VEGFR2 had a significant reduction in the mean ( \pm SEM) area of choroidal NV, and compared with eyes injected with PBS, those injected with aflibercept (anti-VEGF) had a significant reduction in the mean $( \pm$ SEM) area of choroidal NV (G). ${ }^{*} P<0.05,{ }^{* *} P<$ 0.0001 by ANOVA with Bonferroni's correction for multiple comparisons. 
A

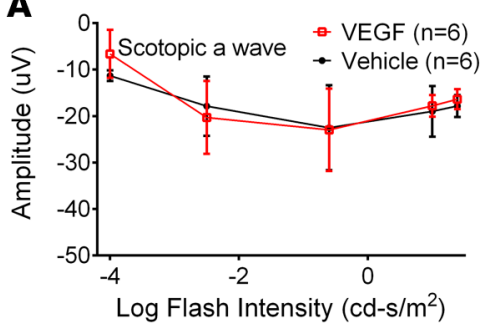

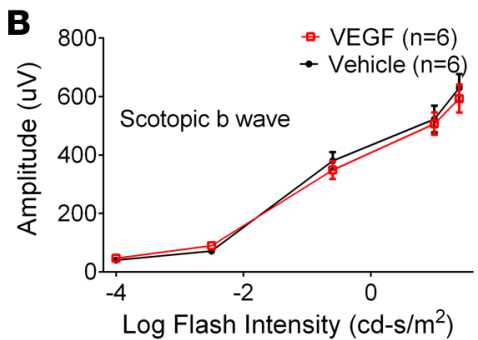

D

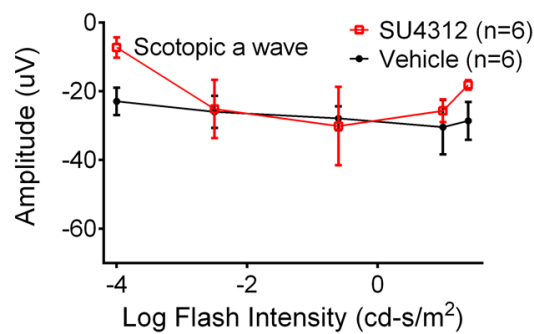

E

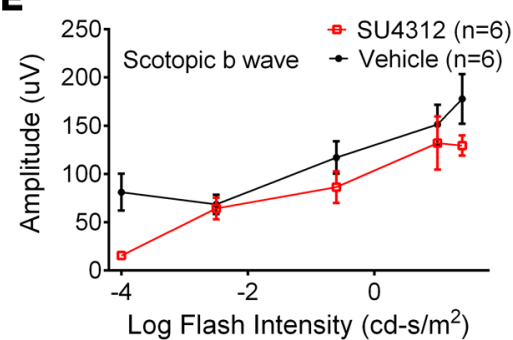

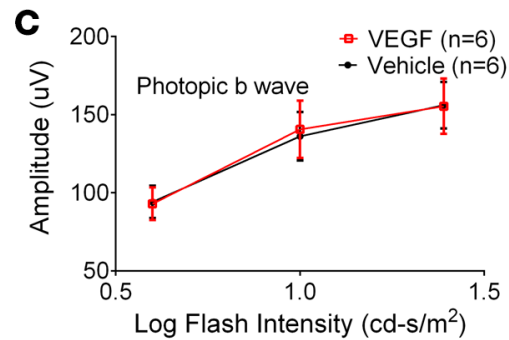

$F$

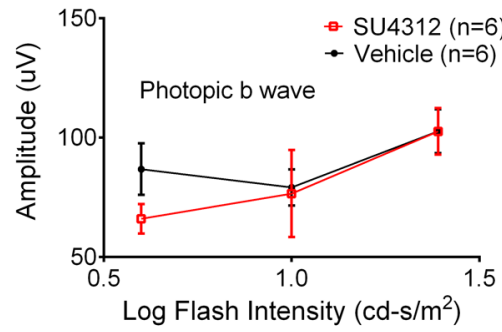

G

Vehicle VEGF

GRK1

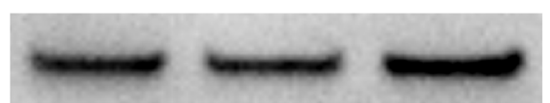

Actin
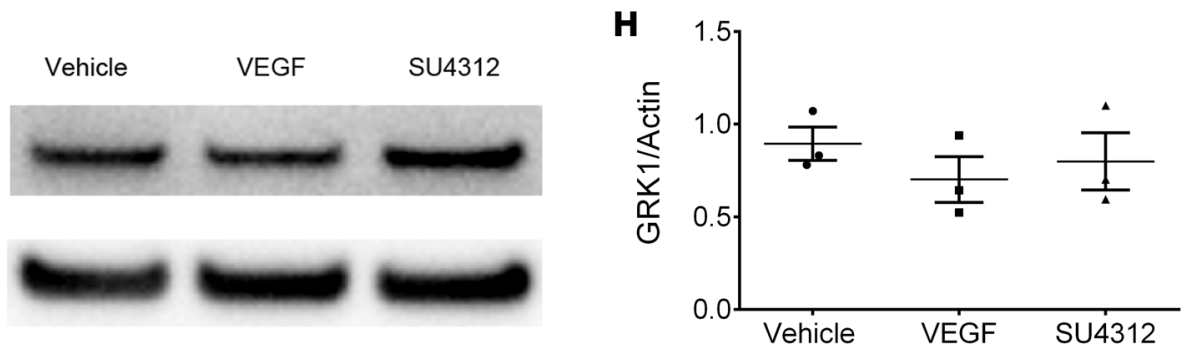

Figure 2. Effect of VEGF or VEGFR blockade on photoreceptor function and survival in rd10 mice with inherited retinal degeneration. Rd10 mice were given an intravitreous injection of 100 ng of VEGF $(n=6)$ or PBS $(n=6)$ on P14, P21, and P28 or they were given subcutaneous injections of $5 \mu$ g of the VEGFR tyrosine kinase inhibitor SU4312 $(n=6)$ or vehicle $(n=6)$ every 5 days starting at P14. At P35, electroretinography showed that compared with eyes injected with vehicle control, those injected with VEGF had no significant differences in mean ( \pm SEM) scotopic a-wave amplitudes (A), mean $( \pm$ SEM) scotopic b-wave amplitudes (B), or mean $( \pm$ SEM) photopic b-wave amplitudes (C). Compared with mice given subcutaneous vehicle injections, those given injections of SU4312 had no significant differences in mean ( \pm SEM) scotopic a-wave amplitudes (D), mean ( \pm SEM) scotopic b-wave amplitudes $(\mathbf{E})$, or mean $( \pm$ SEM) photopic b-wave amplitudes $(\mathbf{F})$. At P35, mice were euthanized and retinal homogenates were immunoblotted for rhodopsin kinase (GRK1) and actin. The bands for GRK1 from vehicle-injected control eyes, VEGF-injected eyes, and eyes from mice treated with SU4312 appeared very similar (G). The mean ( \pm SEM) ratio of GRK1/actin densitometry readings were not significantly different between VEGF $(n=3)$ or SU4312 $(n=3)$ and vehicle controls $(n=3)$ by ANOVA with Bonferroni's correction for multiple comparisons $(\mathbf{H})$.

consider other potential reasons why type 3 choroidal $\mathrm{NV}$ is more prone to retinal atrophy. Measurements of tissue oxygen levels in the retina using oxygen electrodes showed high levels in the choroid that drop precipitously at the level of photoreceptor inner segments that are packed with mitochondria, and then remain low throughout the ONL $(28,29)$. However, type 3 choroidal NV grows through the ONL and provides an additional source of oxygen that would be highest in photoreceptors adjacent to new vessels. High levels of oxygen promote oxidative damage and therefore we hypothesized that increased incidence of photoreceptor cell damage and retinal atrophy in retinas with type 3 choroidal NV is not due to greater susceptibility to VEGF suppression, but rather oxidative damage. To test this hypothesis, we used a mouse model of type 3 choroidal NV, rho/VEGF transgenic mice, in which the rhodopsin promoter drives expression of VEGF in photoreceptors, causing type 3 choroidal NV that originates from the deep capillary bed and grows into the subretinal space $(7,30)$. The retinas of rho/VEGF transgenic mice were stained for 4-hydroxynonenal (HNE), a major aldehydic product of lipid peroxidation $(31,32)$ that has proven to be a useful biomarker of oxidative damage to lipids in several disease processes including retinal disease (33). Compared with wildtype mice, there was increased staining for HNE in rho/VEGF mice at P21 that was located along the outer border of the ONL (Figure 3A) where new vessels stained with Griffonia simplicifolia agglutinin (GSA) lectin were also seen. The HNE staining increased at P28 and P56 particularly along the outer border of the ONL 
and was associated with new vessels in the subretinal space, but there was also some staining in the inner nuclear layer. $\mathrm{N}$-acetylcysteine (NAC) is a potent antioxidant that is bioavailable to the retina after addition to the drinking water. Addition of $7 \mathrm{mg} / \mathrm{ml}$ of NAC to drinking water has been shown to markedly reduce oxidative damage in photoreceptors (34), and this same dose in rho/VEGF mice caused a marked reduction of HNE staining in the retina at P21, P28, and P56 (Figure 3A). Peroxynitrite has a strong affinity for tyrosine residues, resulting in 3-nitrotyrosine residues, a specific marker for peroxynitrite-induced nitrosative damage (35). Unlike wild-type mice at P21, P28, and P56, the retinas of rho/VEGF mice showed intense staining for 3-nitrotyrosine predominantly along the outer border of the ONL, but also in the inner retina at P28 and P56, and the staining was markedly reduced by treatment with NAC (Figure 3B). Retinal flat mounts of P56 wild-type or rho/VEGF mice stained with peanut agglutinin to visualize cone matrix sheaths and GSA lectin to visualize NV showed a regular cone mosaic in wild-type mice (Figure 3C) but there was disruption of the mosaic with dropout of cones in regions of NV in rho/VEGF mice (Figure 3C). The - $\mathrm{COOH}$ groups of proteins are particularly vulnerable to free radical attack resulting in carbonyl adducts and a single protein can have multiple adducts increasing the likelihood of dysfunction, aggregation, and complete loss of function (36). Carbonyl adducts are specific for oxidative damage and their measurement provides a quantitative assessment of the amount of oxidative damage in a particular tissue (37). Measurement of the carbonyl content on proteins in retinal homogenates by ELISA showed that compared with retinas from untreated P56 rho/VEGF mice, those treated with $7 \mathrm{mg} / \mathrm{ml}$ NAC in drinking water between P21 and P56 showed significantly less carbonyl content on retinal proteins (Figure 3D). Measurement of ONL thickness at 6 locations along the vertical meridian, an assessment of rod photoreceptor number, showed a significant reduction in thickness at 4 locations in rho/VEGF mice compared with wild-type mice that was restored to normal in NAC-treated rho/VEGF mice (Figure 3E). Immunoblots for GRK1 of retinal homogenates from P56 rho/VEGF mice showed that treatment with NAC between P21 and P56 resulted in a significant increase in the GSK1/actin ratio (Figure 3F). These data indicate that there is substantial rod and cone cell death due to oxidative damage in rho/VEGF transgenic mice that is prevented by NAC.

Rho/VEGF transgenic mice with type 3 choroidal NV develop a GA phenotype that is prevented by NAC. Fundus photographs of P35 rho/VEGF mice showed numerous hypopigmented spots similar in appearance to those seen on fundus photographs of patients with AMD and GA (Figure 4A). Treatment of rho/VEGF mice with $7 \mathrm{mg} / \mathrm{ml}$ NAC in drinking water between P21 and P35 reduced the appearance of hypopigmented spots in the retina (Figure 4B). Measurement of the area of hypopigmented, atrophic spots per total retinal area by a masked investigator showed that P35 NAC-treated rho/VEGF mice had a significant reduction compared with untreated $\mathrm{P} 35$ rho/VEGF mice (Figure 4C).

Suppression of VEGF does not increase retinal atrophy in mice with type 3 choroidal $N V$. Rho/VEGF mice were given intravitreous injections of $40 \mu \mathrm{g}$ of aflibercept or vehicle at P14, P21, and P28 and at P35, and fundus photographs of 2 representative eyes per group appeared to show fewer atrophic spots in the afliberceptinjected eyes compared with control eyes (Figure 5A). However, image analysis by an investigator masked with respect to treatment group showed a trend toward reduced area of hypopigmented, atrophic spots per total retinal area in aflibercept-injected compared with control eyes, but the difference was not statistically significant (Figure 5D). It is clear, however, that suppression of VEGF did not cause worsening of retinal atrophy. Mice were euthanized and frozen ocular sections were stained with GSA lectin, which stains vascular cells, and counterstained with DAPI to show cell nuclei. Representative retinal sections showed less $\mathrm{NV}$ in the subretinal space than that seen in subretinal space of aflibercept-injected eyes compared with controls (Figure 5, A-C), and while the NV in close proximity to photoreceptor cells bodies was reduced (arrows), it was not eliminated, which is consistent with occurrence of retinal atrophy in aflibercept-injected eyes. The area of subretinal NV was measured on 5 sections, $100 \mu \mathrm{m}$ apart on each side of the optic nerve by a masked investigator and the mean area of subretinal NV per 10 sections was significantly less in aflibercept-injected eyes compared with controls (Figure 5E).

\section{Discussion}

VEGF is a survival factor for endothelial cells in newly formed vessels, but endothelial cells of mature vessels receive other survival signals that originate from extracellular matrix (ECM) and surrounding cells (38). Thus, most mature blood vessels are not compromised by VEGF suppression, but fenestrated capillaries are more susceptible (39). The susceptibility of fenestrated capillaries differs among vascular beds and while the choriocapillaris is fenestrated, it is not adversely affected by systemic suppression of VEGF 

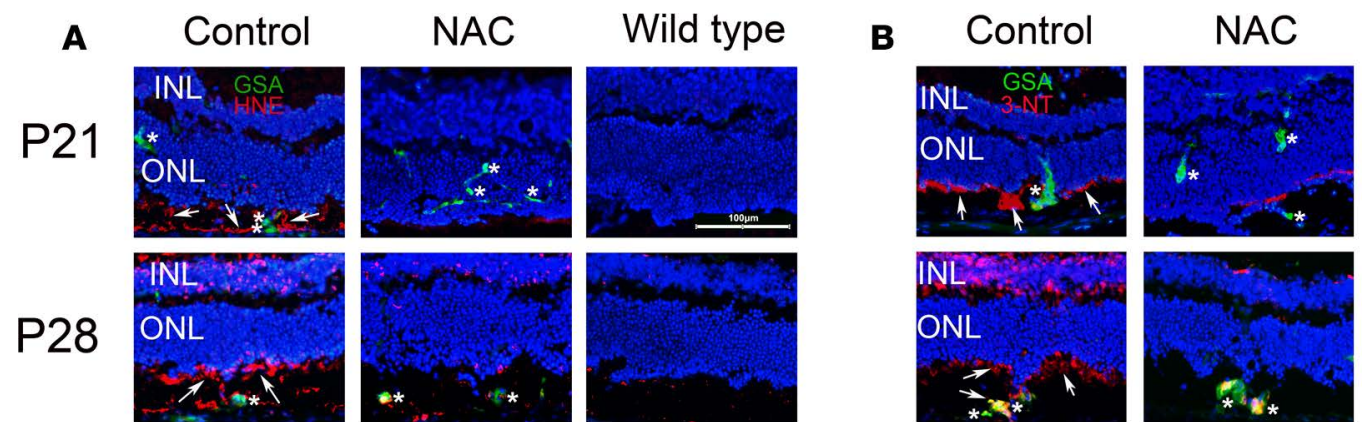

Wild type
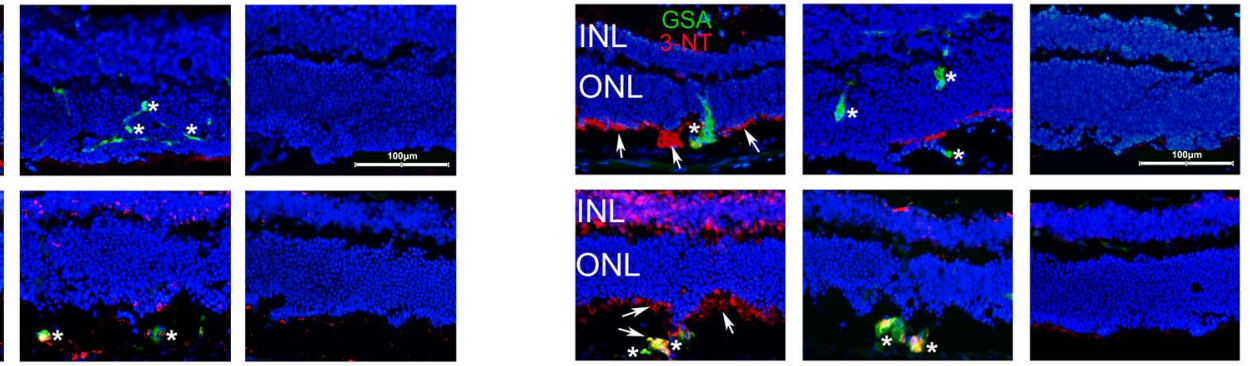

P56
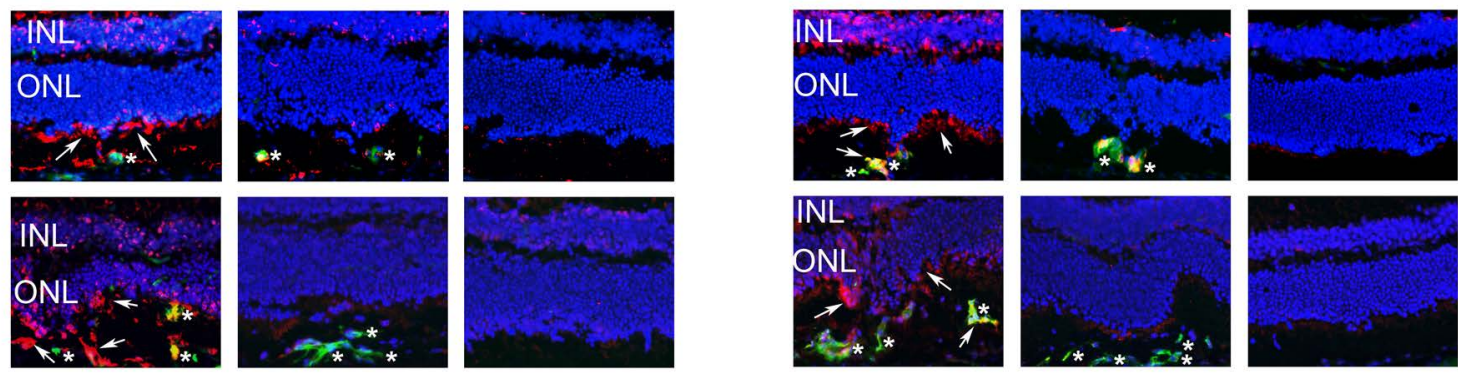

C
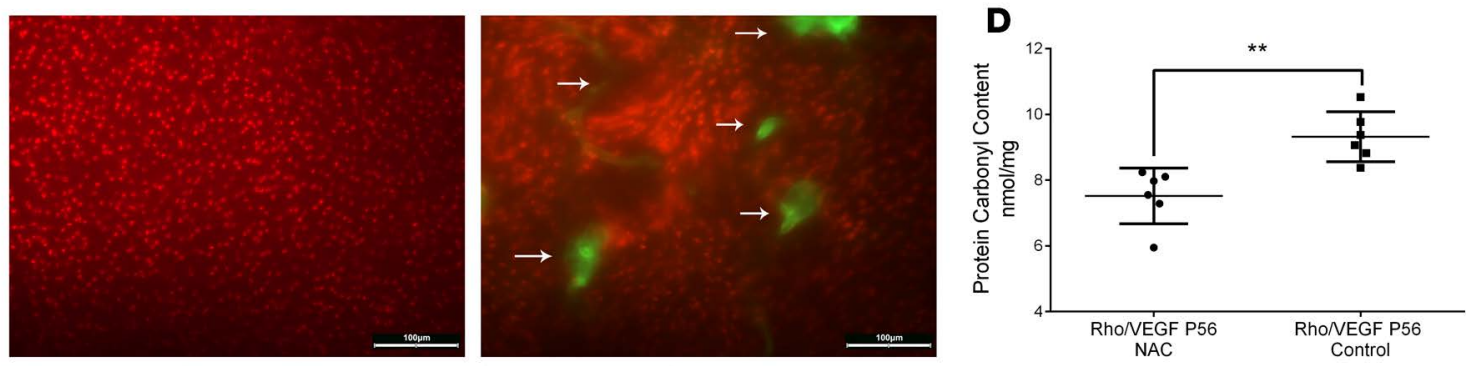

$\mathbf{E}$
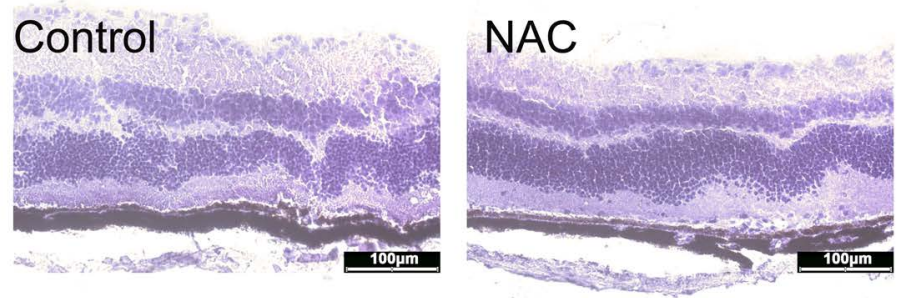

$\mathbf{F}$
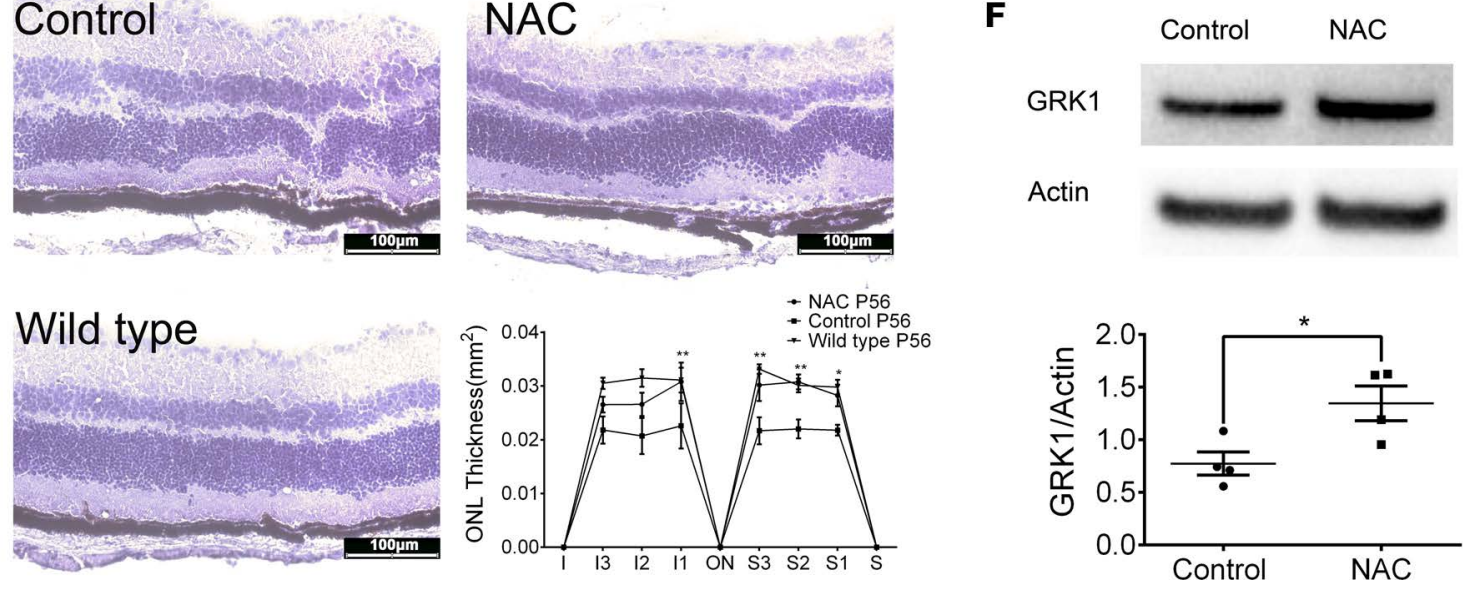

Figure 3. Type 3 choroidal neovascularization (NV) causes photoreceptor cell death from oxidative damage. Rho/VEGF mice with type 3 choroidal NV were given normal drinking water or drinking water containing $7 \mathrm{mg} / \mathrm{ml} \mathrm{N}$-acetylcysteine (NAC) and compared with wild-type mice. At postnatal day 21 (P21), P28, and P56, mice from each of these 3 groups were euthanized and frozen ocular sections were immunohistochemically stained for 4-hydroxynonenal (HNE, A) or 3-nitrotyrosine (3-NT, B) and vascular cells were stained with FITC-labeled Griffonia simplicifolia agglutinin (GSA) lectin. Nuclei were stained with Hoechst. (A) At P21, untreated rho/VEGF mice (control) showed HNE (red, arrows) along the outer border of the outer nuclear layer (ONL) and in the subretinal space adjacent to GSA-stained NV (green, asterisks), which was increased at P28 and P56 when there was also staining within the ONL and inner nuclear layer (INL). In NAC-treated rho/VECF mice there was minimal HNE at each of the time points but NV (green, asterisks) was seen within the ONL and subretinal space. At P28, some mild staining for HNE was seen adjacent to and within NV in the subretinal space. There was no HNE or NV seen in wild-type mice. (B) Control rho/VEGF mice also showed 3-NT along the outer border of the ONL (red, arrows) adjacent to NV (green, asterisks) at all 3 time points with some staining in the INL at the later time points, whereas there was little 3-NT in NAC-treated rho/VECF mice, and none in wild-type mice. (C) Retinal flat mounts stained with peanut agglutinin (red) and CSA (green) showed evenly spaced cone matrix sheaths and no NV in P56 wild-type mice (left panel), whereas P56 rho/VEGF mice (right panel) showed dropout of cone matrix sheaths adjacent to NV (arrows). (D) ELISA of retinal homogenates showed a significant reduction in mean ( \pm SEM) protein carbonyl content in NAC-treated $r$ ho/VEGF mice compared with control rho/VEGF mice $\left(n=6\right.$ for each group). ${ }^{* *} P<0.01$ by unpaired $t$ test. (E) Hematoxylin-stained retinal sections from the same location in the retina (I3) show an ONL with irregular borders in untreated (control) and NAC-treated P56 rho/VEGF mice (NAC), but the ONL appeared thicker in NAC-treated mice and more comparable to the ONL in P56 wild-type mice. Image analysis by an investigator masked with regard to treatment group showed that mean $( \pm$ SEM) ONL thickness was significantly reduced in control versus NAC-treated rho/VEGF mice at 4 of 6 measurement locations in the vertical meridian ( $n=6$ for each group). ${ }^{*} P<0.05,{ }^{* *} P<0.01$ by unpaired $t$ test. For context, mean $( \pm$ SEM) ONL thickness at the same 6 locations is shown for P56 wild-type mice $(n=6)$. (F) A representative immunoblot for rhodopsin kinase (GRK1) shows a stronger signal in a retinal homogenate from a P56 NAC-treated rho/VEGF mouse compared with an untreated P56 rho/VEGF mouse (control). Densitometry showed that the mean ( \pm SEM) GRK1/actin ratio was significantly greater in NAC-treated mice $(n=4)$. ${ }^{*} P<0.05$ by unpaired $t$ test. 
A

Control P35 Rho/VEGF
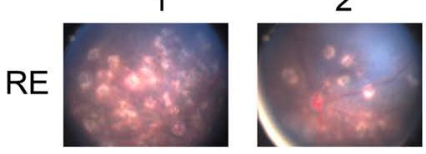

LE
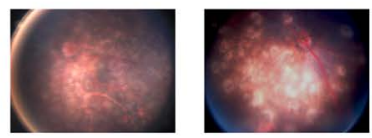

B

NAC P35 Rho/VEGF
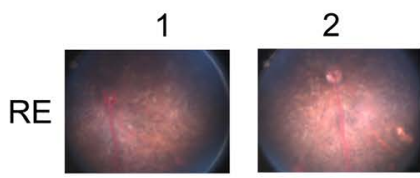

LE
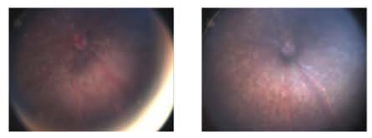

3

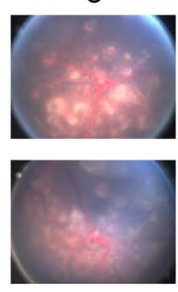

3
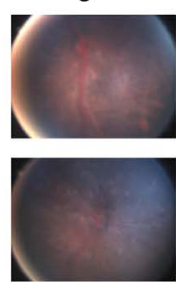

C

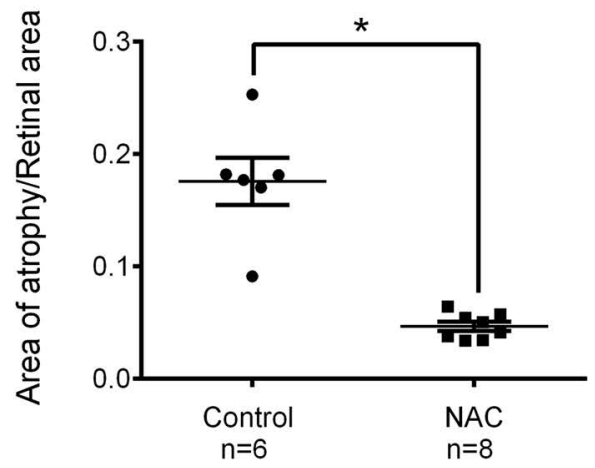

Figure 4. Rho/VEGF mice with type 3 choroidal neovascularization have a geographic atrophy phenotype that is reduced by treatment with $\mathbf{N}$-acetylcysteine (NAC). (A) Fundus photographs of the right eye (RE) and left eye (LE) of 3 representative untreated (control) P35 rho/VEGF mice show numerous hypopigmented spots. (B) Fundus photographs of the RE and LE of NAC-treated P35 rho/VEGF mice show few small hypopigmented spots. (C) Image analysis with investigator masked with respect to treatment group showed a statistically significant reduction in mean ( \pm SEM) ratio of atrophic area/retinal area in NAC-treated rho/VEGF mice versus control rho/VECF mice. ${ }^{*} P<0.0001$ by unpaired $t$ test.

in oncology patients, while some of the fenestrated capillaries of the thyroid and trachea undergo reversible regression (40). However, conditional knockout of VEGF in the RPE causes rapid regression of the choriocapillaris and overlying retina, indicating dependence of choriocapillaris on paracrine VEGF from RPE (12), but it appears unlikely that the paracrine delivery of VEGF between RPE and choriocapillaris can be disrupted by biologics or drugs that suppress VEGF, because high-level retinal expression of a potent VEGF-neutralizing protein for up to 7 months had no effect on the choriocapillaris or the retina (13).

Endothelial cells of choroidal NV in patients with NVAMD are fenestrated but they lose dependence on VEGF for survival relatively soon after formation, because intraocular injections of a VEGF-neutralizing protein reduced leakage from the choroidal $\mathrm{NV}$ and improved vision, but the mean area of choroidal $\mathrm{NV}$ measured on fluorescein angiograms was not reduced over the course of 2 years (8). This explains the need for repeated injections of a VEGF-neutralizing protein in patients with NVAMD, because they do not eliminate choroidal NV and as intraocular levels of the antagonist decline, levels of VEGF increase and stimulate the choroidal NV to resume leakage, growth, recruitment of other cells, and deposition of ECM. If retreatment is delayed, recruitment of cells and deposition of ECM around choroidal NV promotes scarring, damage to photoreceptors, and permanent loss of vision. Whether brief periods of VEGF elevation between periods of VEGF suppression are harmful is uncertain, but the demonstration that compared with PRN injections of a VEGF-neutralizing protein, which allow frequent albeit brief recurrences, monthly injections provide superior visual outcomes over the span of 2 years (15), suggests that they may.

While the benefits of VEGF suppression are impressive and undisputed in the treatment of retinal and choroidal vascular diseases (41), there is still considerable debate regarding the risks, because it has been hypothesized that VEGF promotes survival of retinal photoreceptors as well as retinal vascular endothelial cells (11). The current study was designed to test that hypothesis and the data suggest that VEGF does not promote functioning or survival of photoreceptors. The specific findings that support this conclusion are the following. (a) Intraocular injection of an antibody that blocks VEGFR2 strongly suppressed choroidal NV at Bruch's membrane rupture sites in rats, but caused no significant reduction in photoreceptor ERG function or evidence of photoreceptor cell death. (b) Intraocular injections of VEGF did not slow the loss of photoreceptor ERG function or photoreceptor cell death in $r d 10$ mice with inherited retinal degeneration, as has been demonstrated for other proteins that provide neurotrophic support for photoreceptors $(18,19)$. (c) Blockade of VEGFR signaling did not accelerate loss of photoreceptor ERG function or photoreceptor cell death in $r d 10$ mice with inherited retinal degeneration. These data suggest that suppression of VEGF is not likely to 
A
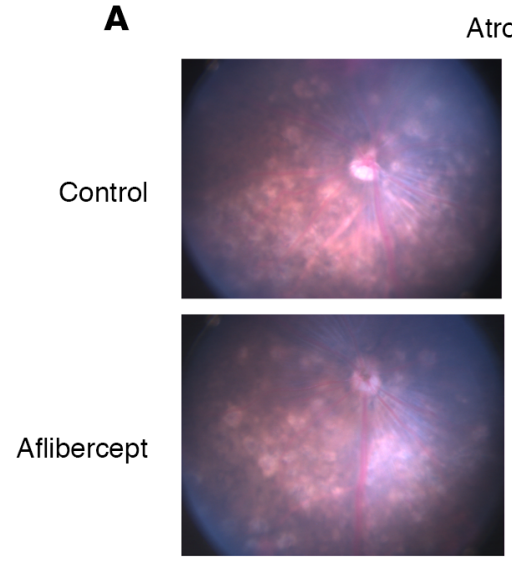

$\mathbf{B}$

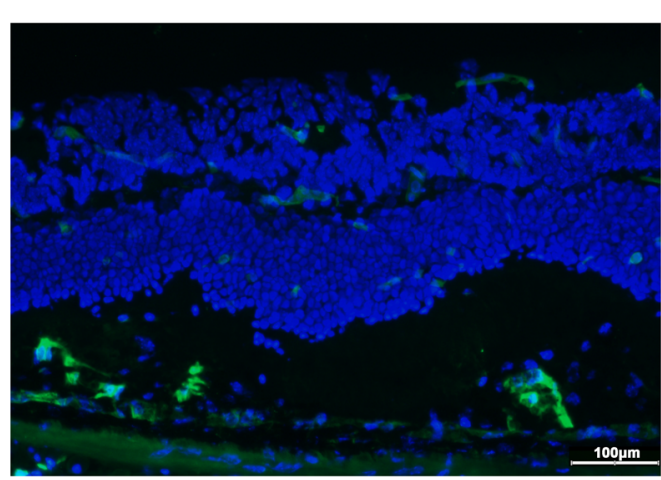

c

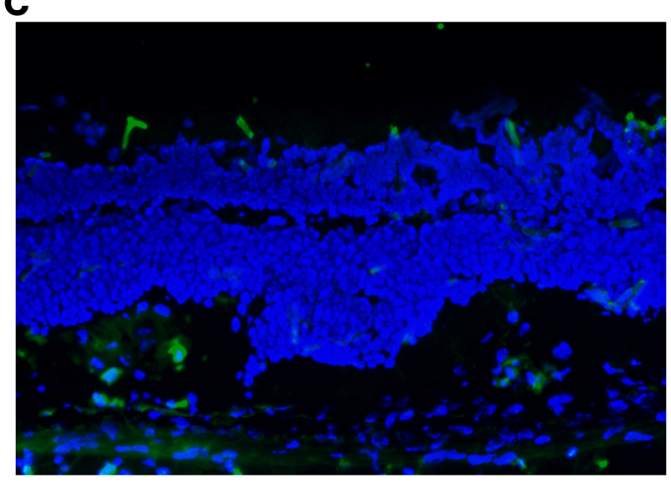

E
D

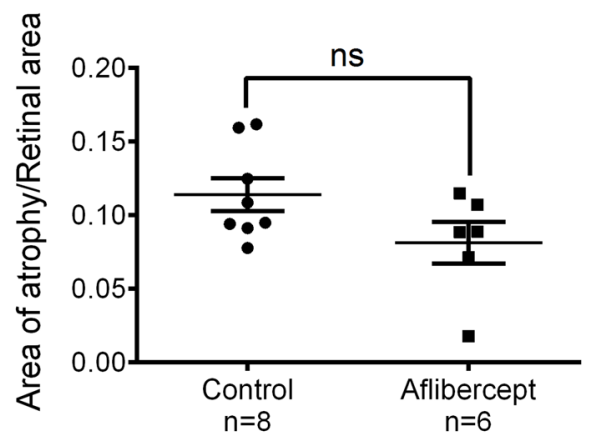

Neovascularization
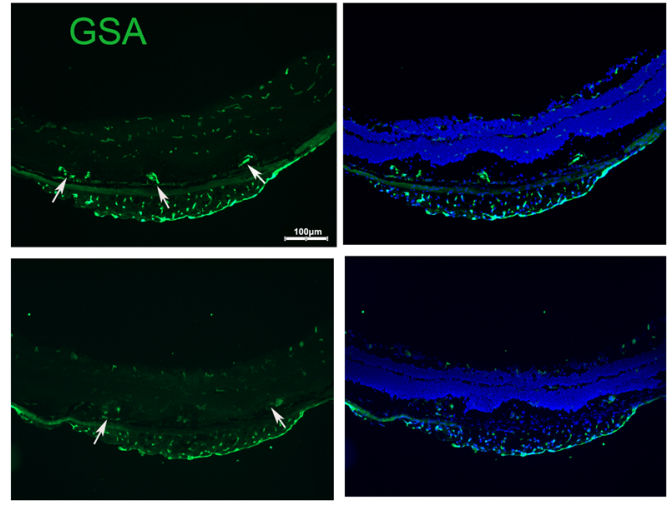
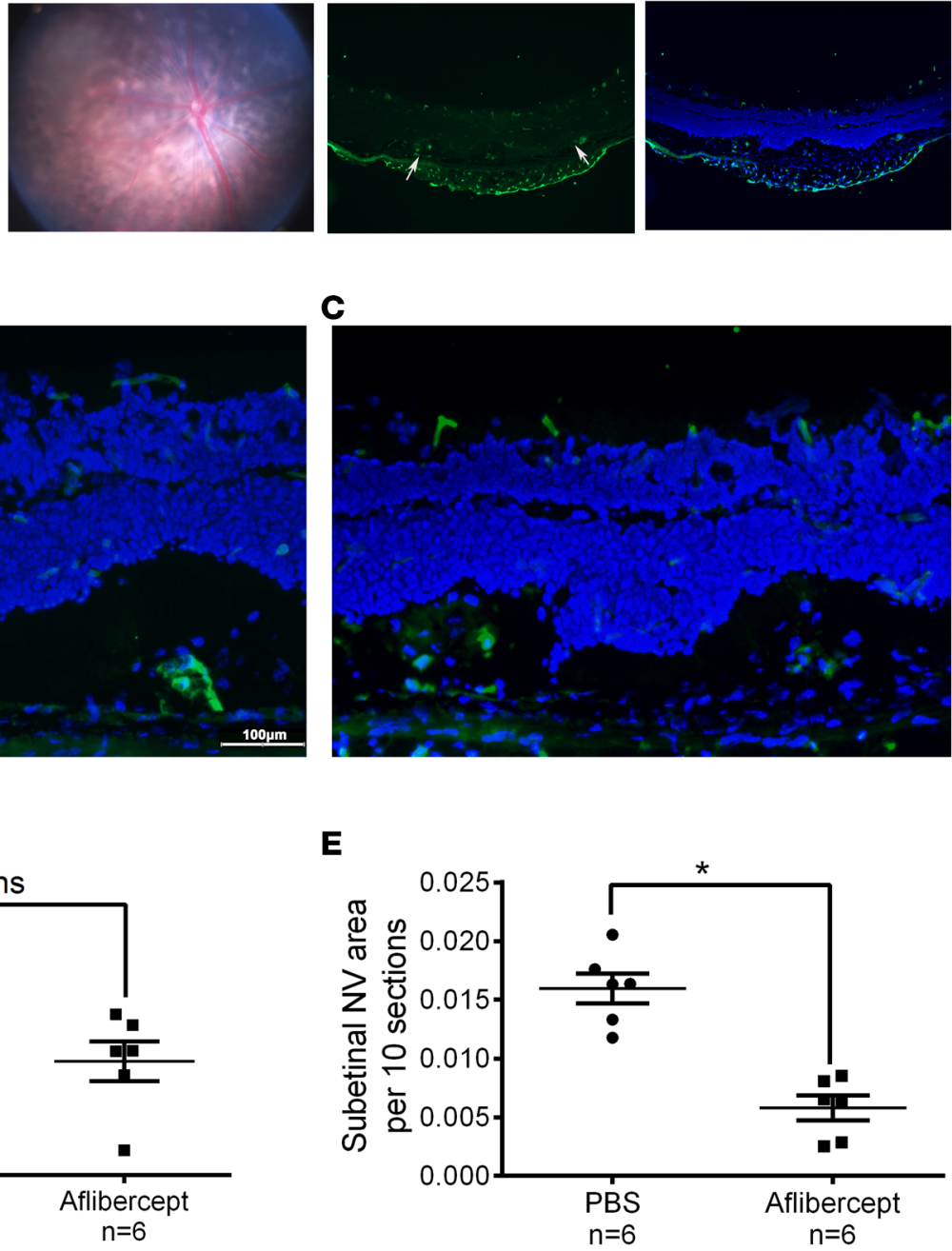

Figure 5. Retinal atrophy in rho/VEGF mice with type 3 choroidal neovascularization is not increased by suppression of VEGF by aflibercept. (A) Fundus photographs (columns 1 and 2) of 2 representative eyes from untreated (control) and aflibercept-treated P35 rho/VEGF mice show numerous hypopigmented spots with the appearance of somewhat fewer in aflibercept-treated eyes. Frozen sections stained with Griffonia simplicifolia agglutinin (GSA, green) lectin alone (column 3 ) or with nuclei of retinal cells counterstained with Hoechst (blue, column 4) show less neovascularization (NV) in the subretinal space and in close proximity to the outer nuclear layer (arrows) in aflibercept-treated eyes more easily seen in high-magnification images of control (B) and aflibercepttreated (C) eyes. Image analysis with investigator masked with respect to treatment group showed no statistically significant difference $(P=0.0933$ by Mann-Whitney $U$ test) in mean ( \pm SEM) ratio of atrophic area/retinal area in aflibercept-treated rho/VEGF mice compared with control rho/VEGF mice (D), but the aflibercept-treated mice had significantly less subretinal NV (E). ${ }^{*} P<0.01$ by Mann-Whitney $U$ test. ns, not significant.

contribute to retinal atrophy in patients with NVAMD. This is also suggested by the clinical observations that retinal atrophy only occurs in patients with NVAMD treated with anti-VEGF agents and not in patients with diabetic macular edema or macular edema due to retinal vein occlusion treated with equivalent VEGF suppression. A preclinical study suggesting that VEGF suppression causes retinal degeneration in diabetic mice (42) is inconsistent with findings in clinical trials (43). In fact, it has been shown that monthly injections of ranibizumab reverse diabetic retinopathy and the safety profile is so good that the FDA modified the label for ranibizumab to include patients with completely normal vision who have background diabetic retinopathy. 
Among patients with NVAMD treated with anti-VEGF agents, retinal atrophy is more common in patients with type 3 choroidal NV compared with those with type 1 or 2 choroidal NV. In type 3 choroidal NV, new vessels pass through the ONL and are in close association with photoreceptor cell bodies as well as inner and outer segments, whereas type 1 choroidal NV remains beneath the RPE, remote from photoreceptors, and type 2 choroidal NV is in the subretinal space in close association with photoreceptor outer segments but not other parts of the cell. We hypothesized that the close association of type 3 choroidal NV with photoreceptors is a source of oxidative damage and this hypothesis was found to be correct, because mice with type 3 choroidal NV showed progressive oxidative damage to photoreceptors, photoreceptor cell death, and hypopigmented spots typical of retinal atrophy and all of these were significantly reduced by treatment with the potent antioxidant NAC. VEGF suppression did not worsen the retinal atrophy in mice with type 3 choroidal NV. Thus, retinal atrophy in the setting of type 3 choroidal NV occurs due to oxidative damage and is not worsened by VEGF suppression.

If VEGF does not provide trophic support to photoreceptors, what is the function of VEGFR2 on retinal neurons? Genetic deletion of VEGFR2 in neurons caused abnormal vessel growth toward retinal neurons resulting in abnormal vessel density around neurons (44). The misdirected vessel growth was due to increased levels of VEGF around retinal neurons, because lack of VEGFR2 on neurons reduced VEGF internalization and degradation. Thus, VEGFR2 on neurons provides reverse VEGF gradients that reduce growth of vessels around neurons. Mice deficient in VEGFR2 had normal development and survival of all retinal neurons and adults showed normal morphology and function, indicating that VEGFR2 is not required for normal survival and functioning of retinal neurons.

These data have important clinical implications because concern that VEGF suppression is damaging to retinal neurons and a potential cause of retinal atrophy in patients with NVAMD treated with antiVEGF injections has led to recommendations to avoid frequent injections $(15,16)$. At the 2-year endpoint of the CATT trial, subjects in all 4 of the anti-VEGF treatment arms showed large mean improvements in visual acuity from baseline. During the next 3 years, patients received fewer mean number of injections as part of standard care and for the population as a whole, all of the visual gains achieved during the first 2 years were lost (45). While there are a number of possible causes for this loss of vision, it appears that insufficient treatment may have contributed because (a) the goal of treatment is to eliminate fluid within or under the macula, but fluid was present in $83 \%$ of 555 eyes in which spectral domain OCT was obtained, a very large percentage; (b) intraretinal fluid is particularly problematic because it is a risk factor for poor outcome and more eyes had intraretinal fluid at 5 years than at 2 years (61\% versus $50 \%$ ); and (c) VEGF suppression prevents growth of choroidal NV, but in 467 eyes in which fluorescein angiography was done at 5 years, total lesion area was $4.8 \mathrm{~mm}^{2}$ larger than at 2 years, an increase of more than $50 \%$, indicating that there had been poor VEGF suppression. In contrast, in a study in which 171 patients with NVAMD received frequent injections for 5 years (average of 10.4 per year for 5 years), the mean improvement from baseline at 5 years was 14.1 letters (46). While caution should be used when comparing outcomes in different studies because of possible differences in study populations, a gain of 14.1 letters at 5 years versus a loss of 3.3 letters from baseline at 5 years in CATT is such an enormous difference that it is difficult to ignore.

A large observational study of 2,227 NVAMD patients with anti-VEGF treatment in clinical practice found that patients received a mean of 5.0 and 2.2 injections in the first and second year and mean improvement in visual acuity score was +2.4 letters at year 1 and +0.6 letters at year 2 (47). The number of anti-VEGF injections administered was much lower than in clinical trials and visual outcome was far inferior. Concern that frequent anti-VEGF injections may lead to retinal atrophy may be contributing to insufficient treatment and poor outcomes in clinical practice and it is important to allay the fears of clinicians and raise awareness of potential hazards of insufficient treatment. New approaches to achieve sustained suppression of VEGF are being tested including implantation of a refillable reservoir that slowly releases ranibizumab into the eye (ClinicalTrials.gov identifier: NCT02510794) and gene transfer to express VEGFneutralizing proteins in the eye (48), and they should provide more information regarding the effect of sustained suppression of VEGF on retinal function. Subretinal injection of adeno-associated virus (AAV) vectors expressing a VEGF-binding protein will be a particularly stringent test, because AAV vectors cause high-level transgene expression in RPE cells and when the transgene is a potent VEGF-binding protein, it is conceivable that this could mimic genetic deletion of VEGF. While the current study does not guarantee safety of highly efficient knockdown of VEGF in RPE cells, it should reduce fear of direct damage to retinal neurons from VEGF suppression, eliminate concerns of retinal atrophy from intravitreous injections of VEGF, and encourage clinicians to treat patients with NVAMD by aggressive suppression of VEGF. 


\section{Methods}

Testing effects of anti-VEGFR 2 in rats. Brown Norway rats were obtained from Charles River Laboratories. To test whether suppression of VEGFR2 causes reduced photoreceptor cell function and death, 3 intraocular injections a week apart of $40 \mu \mathrm{g}$ of an antibody directed against human VEGFR2 that cross-reacts with rat VEGFR2 (anti-KDR B1A1, Kadmon Corporation) were given in one eye and $40 \mu \mathrm{g}$ of control IgG (EquitecBio Inc., SLH56-0001) was injected in the fellow eye of Brown Norway rats. One week after the third injection of anti-VEGFR2, ERGs were recorded. The next day, fundus photographs and OCT scans were done and rats were euthanized for measurement of ONL thickness.

In order to assess the effect of suppression of VEGFR2 on choroidal NV, Brown Norway rats were anesthetized, pupils were dilated with 1\% tropicamide (Alcon Laboratories Inc.), and Bruch's membrane was ruptured at the $3,6,9$, and 12 o'clock positions of the posterior pole (100- $\mu \mathrm{m}$ spot size, 0.1 -second duration, $120 \mathrm{~mW}$ ) using the slit lamp delivery system of a 532-nm diode OcuLight GL Photocoagulator (Iridex) and a handheld cover slide as a contact lens. Rats then had intraocular injection of $20 \mu \mathrm{g}$ antiVEGFR2 or control IgG, $20 \mu \mathrm{g}$ of the VEGF-neutralizing protein aflibercept (Regeneron), or PBS. After 14 days, rats were euthanized, eyes were removed, retinas were dissected, and eye cups, consisting of RPE, choroid, and sclera were stained with FITC-labeled GSA lectin, and flat-mounted. Flat mounts were examined by fluorescence microscopy, and the area of choroidal NV at each Bruch's membrane rupture site was measured by image analysis with Image-Pro Plus software (Media Cybernetics) by an observer masked with respect to experimental groups.

Testing effects of VEGF and VEGF suppression in mice with inherited retinal degeneration. Rd10 mice with inherited retinal degeneration were obtained from Jackson Labs. They were given an intraocular injection of $100 \mathrm{ng}$ of $\mathrm{VEGF}_{165}$ (R\&D Systems) or PBS on P14, P21, P28, P35, or a subcutaneous injection of $5 \mu \mathrm{g}$ of the VEGFR tyrosine kinase inhibitor SU4312 (Sigma-Aldrich) or vehicle every 5 days starting at P14. Fundus photographs and ERGs were done at P35 and then mice were euthanized, eyes were removed, retinas were dissected, and immunoblots for rhodopsin kinase were done on retinal homogenates.

Testing for oxidative damage and photoreceptor cell degeneration in rho/VEGF mice with type 3 choroidal $N V$. The generation and characterization of transgenic mice in which the rhodopsin promoter drives expression of VEGF in photoreceptors (rho/VEGF mice) have been described (7, 30). Untreated rho/ VEGF mice and wild-type C57BL/6J mice and those treated with $7 \mathrm{mg} / \mathrm{ml}$ NAC (Sigma-Aldrich) in drinking water starting at P14 were euthanized at P14, P21, P28, and P56 ( $n=10$ for each time point). This dose of NAC was selected because it had previously been demonstrated to effectively suppress oxidative damage in the retina (34). One eye was used to measure carbonyl content on retinal proteins by ELISA and the other eye was used for immunohistochemistry for HNE or 3-nitrotyrosine. Another group of $r h o / V E G F$ mice were given $7 \mathrm{mg} / \mathrm{ml}$ NAC in drinking water or were treated with subcutaneous injections of $5 \mu \mathrm{g}$ of SU4312 or vehicle every 5 days starting at P14. At P35, fundus photos were taken to assess for retinal atrophy and then immunoblots for rhodopsin kinase and actin were done on retinal homogenates.

ERG recordings. ERGs were recorded with an Espion ERG Diagnosys machine (Diagnosys) as previously described (49). Briefly, rats or mice were anesthetized by intraperitoneal injection of ketamine $(100 \mathrm{mg} / \mathrm{kg})$ and xylazine $(5 \mathrm{mg} / \mathrm{kg})$, placed on a pad heated to $39^{\circ} \mathrm{C}$, pupils were dilated, and platinum loop electrodes were placed on each cornea after application of gonioscopic prism solution (Alcon Laboratories Inc.). A reference electrode was placed subcutaneously in the anterior scalp between the eyes and a ground electrode was inserted into the base of the tail. The head was held in a standardized position in a ganzfeld bowl illuminator that ensured equal illumination of the eyes. For scotopic recordings, animals were dark adapted overnight and ERG waveforms were recorded at 11 flash intensities $\left(-4,-2.5,-2.2,-1.79,-1.39,-1,-0.6,-0.39,-0.20,1.0,1.39 \mathrm{log} \mathrm{cd} \cdot \mathrm{s} / \mathrm{m}^{2}\right)$. For each flash intensity, 5 readings were taken and averaged. For photopic ERGs, animals were adapted for 7 minutes to a background of white light at an intensity of $30 \mathrm{cdm}^{2}$ and ERG wave forms were recorded after light flashes at 3 intensities $\left(0.6,1.0,1.39 \mathrm{log} \mathrm{cd} \cdot \mathrm{s} / \mathrm{m}^{2}\right)$. For each flash intensity, 5 readings were taken and averaged.

Fundus photographs and OCT. Fundus photographs were obtained with a Micron III Retinal Imaging Microscope (Phoenix Research Laboratories Inc.). The area of retinal atrophy per total area of retina was measured on fundus photographs of rho/VEGF mice by image analysis with Image-Pro Plus software with the investigator masked with respect to treatment group. OCT was done using a Bioptigen SD-OCT ophthalmic imaging system XHR 4110 (Leica Microsystems Inc.). 
Measurement of ONL thickness. The thickness of the ONL, which correlates with rod cell number in the retina, was measured as previously described (50). Mice were euthanized, a mark was placed at 12:00 at the corneal limbus, and eyes were removed and frozen in optimum cutting temperature embedding compound (Miles Diagnostics). Ten-micrometer frozen sections were cut parallel to 12:00 meridian through the optic nerve and fixed in $4 \%$ paraformaldehyde. The sections were stained with hematoxylin, examined with an Axioskop microscope (Zeiss) and digital images obtained. Image-Pro Plus software was used to outline the ONL. With the observer masked with respect to treatment group, ONL thickness was measured at 6 locations, $25 \%$ (S1), $50 \%(\mathrm{~S} 2)$, and $75 \%$ (S3) of the distance between the superior pole and the optic nerve and $25 \%$ (I1), $50 \%$ (I2), and 75\% (I3) of the distance between the inferior pole and the optic nerve.

Measurement of cone cell density. Cone cell density was measured as previously described (49). After removal of the cornea, iris, and lens, a small cut was made at 12:00 in the retina for future orientation. Eyecups were fixed in 4\% paraformaldehyde for 2 hours, and then the entire retina was carefully dissected away from the RPE, severed at the optic nerve, and removed from the eye. Retinas were placed in $10 \%$ normal goat serum in PBS for 30 minutes at $23^{\circ} \mathrm{C}$, incubated for 1 hour at $23^{\circ} \mathrm{C}$ in $1: 30$ rhodamineconjugated peanut agglutinin (PNA; Vector Laboratories, RL-1072) in PBS containing 1\% normal goat serum, and flat-mounted with the photoreceptors facing upward. The retinas were examined with a Zeiss LSM 510 META confocal microscope with a Zeiss Plan-Apochromat 20×/0.75-N.A. objective using an excitation wavelength of $543 \mathrm{~nm}$ to detect rhodamine fluorescence. Retinas were examined by oil immersion with a $63 \times / 1.4-N$.A. Zeiss Plan-Apochromat objective to evaluate cone morphology in detail. Images were acquired in the frame-scan mode. The number of cones present within four $230 \times$ $230 \mu \mathrm{m}(512 \times 512$ pixels $)$ squares located $0.5 \mathrm{~mm}$ superior, temporal, inferior, and nasal to the center of the optic nerve was determined.

Immunohistochemical staining. Ocular frozen sections $(10 \mu \mathrm{m})$ were stained for HNE with rabbit antiHNE (1:100, Alpha Diagnostics International, Inc., HNE11) and 3-nitrotyrosine with rabbit anti-nitrotyrosine (1:100, EMD Millipore Co., 06-284) as previously described (33). Briefly, frozen sections were dried at $23^{\circ} \mathrm{C}$ and postfixed in cold acetone for 15 minutes. Sections were washed with PBS and blocked with $8 \%$ normal goat serum in PBS for 1 hour at $23^{\circ} \mathrm{C}$. The sections were incubated overnight at $4^{\circ} \mathrm{C}$ with one of the above primary antibodies and costained with FITC-labeled GSA (Vector Laboratories, FL-1201). After washing 3 times with PBS $/ 0.05 \%$ Tween 20 , sections were incubated for 1 hour at $23^{\circ} \mathrm{C}$ with a fluorescently labeled secondary antibody. After washing 3 times, slides were counterstained for 3 minutes at $23^{\circ} \mathrm{C}$ with the nuclear dye Hoechst 33258 (1:1,200; Sigma-Aldrich). Slides were examined by fluorescence microscopy and images were obtained using the same exposure time for each section.

Immunoblots for rhodopsin kinase. At P56, mice were euthanized, retinas were dissected from each eye and homogenized in $150 \mu 1$ lysis buffer (Abcam) followed by high-speed centrifugation $(16,000 \mathrm{~g}, 15 \mathrm{~min}$ utes). Supernatants were collected in prechilled Eppendorf tubes. Total protein concentrations were determined using the BCA protein assay kit (Bio-Rad) and samples containing $2 \mu \mathrm{g}$ protein in loading buffer were resolved by $4 \%-12 \%$ Nu-polyacrylamide gel electrophoresis (Invitrogen) and transferred to polyvinylidene difluoride (PVDF) membranes (Invitrogen) and blocked with 5\% blocker milk. Membranes were probed with anti-GRK1 antibody (1:1,000; Abcam, Ab108502) or actin (1:2,000; Cell Signaling Technology, 4970) overnight at $4^{\circ} \mathrm{C}$. HRP-conjugated goat anti-rabbit antibody (1:10,000; Invitrogen, A27036) was added followed by incubation in SuperSignal West Dura Extended Duration Substrate (ThermoFisher Scientific), and Western blots were imaged and analyzed with a Bio-Rad imaging system.

Measurement of carbonyl content on retinal proteins by ELISA. Retinal homogenates in $10 \mathrm{mM}$ Tris, $\mathrm{pH}$ 7.2/0.5\% Triton X-100/50 mM NaCl/1 mM EDTA with a proteinase inhibitor mixture tablet (Roche) were centrifuged at $16,000 \mathrm{~g}$ for 10 minutes at $4^{\circ} \mathrm{C}$. Protein concentrations of supernatants were determined using the BCA kit. Samples were adjusted to $4 \mathrm{mg}$ protein $/ \mathrm{ml}$, and protein carbonyl content was measured using an OxiSelect Protein Carbonyl ELISA Kit (Cell Biolabs Inc.).

Statistics. In experiments in which a single experimental group was compared to a single control group, statistical comparisons were made by unpaired Student's $t$ test. In experiments in which multiple experimental groups were compared to a control group or there were comparisons among multiple experimental groups, comparisons were made by ANOVA with Bonferroni's correction for multiple comparisons.

Study approval. All studies involving rats or mice were approved by the Animal Care and Use Committee of Johns Hopkins University and conformed to the recommendations of the Association for Research in Vision and Ophthalmology and the US NIH Guide for the Care and Use of Laboratory Animals. 


\section{Author contributions}

DL and YK helped to design experiments, performed the majority of experiments, analyzed data, prepared figures, helped to write the first draft, and edited manuscript. JS, SFH, LY, ZH, MK, and LL helped to design and perform some experiments, analyzed data, and edited the manuscript. PAC designed and supervised experiments, analyzed data, and wrote the first draft of the manuscript.

\section{Acknowledgments}

The authors thank Kadmon Corporation for providing the antibody directed against human VEGFR2 that cross-reacts with rat VEGFR2 and Jiangxia Wang of the Johns Hopkins Biostatistics Center, Department of Biostatistics, Johns Hopkins Bloomberg School of Public Health, Baltimore, MD for statistics consultation. This work was supported by Biostatistics Core Grant EY01765 to the Wilmer Eye Institute and unrestricted grants from Andrew and Yvette Marriott, Per Bang-Jensen, Jean Lake, and Conrad and Lois Aschenbach. PAC is the George S. and Dolores Dore Eccles Professor of Ophthalmology and Neuroscience.

Address correspondence to: Peter A. Campochiaro, 815 Maumenee, The Wilmer Eye Institute, Johns Hopkins University School of Medicine, 600 N. Wolfe Street, Baltimore, Maryland, USA. Phone: 410.955.5106; Email: pcampo@jhmi.edu.

DL's present address is: Department of Ophthalmology, The First Hospital of Hunan University of Chinese Medicine, Changsha, China.

YL's present address is: Department of Ophthalmology, Tianjin Medical University General Hospital, Tianjin, China.

1. Klein R, et al. Prevalence of age-related macular degeneration in 4 racial/ethnic groups in the multi-ethnic study of atherosclerosis. Ophthalmology. 2006;113(3):373-380.

2. Curcio CA, Medeiros NE, Millican CL. Photoreceptor loss in age-related macular degeneration. Invest Ophthalmol Vis Sci. 1996;37(7):1236-1249.

3. Fleckenstein M, et al. The Progression of Geographic Atrophy Secondary to Age-Related Macular Degeneration. Ophthalmology. 2018;125(3):369-390.

4. Yannuzzi LA, et al. Retinal angiomatous proliferation in age-related macular degeneration. Retina (Philadelphia, Pa). 2001;21(5):416-434

5. Kwak N, Okamoto N, Wood JM, Campochiaro PA. VEGF is major stimulator in model of choroidal neovascularization. Invest Ophthalmol Vis Sci. 2000;41(10):3158-3164.

6. Krzystolik MG, et al. Prevention of experimental choroidal neovascularization with intravitreal anti-vascular endothelial growth factor antibody fragment. Arch Ophthalmol. 2002;120(3):338-346.

7. Okamoto N, et al. Transgenic mice with increased expression of vascular endothelial growth factor in the retina: a new model of intraretinal and subretinal neovascularization. Am J Pathol. 1997;151(1):281-291.

8. Rosenfeld PJ, et al. Ranibizumab for neovascular age-related macular degeneration. N Engl J Med. 2006;355(14):1419-1431.

9. Brown DM, et al. Ranibizumab versus verteporfin for neovascular age-related macular degeneration. $N$ Engl J Med. 2006;355(14):1432-1444.

10. Singer MA, et al. HORIZON: an open-label extension trial of ranibizumab for choroidal neovascularization secondary to agerelated macular degeneration. Ophthalmology. 2012;119(6):1175-1183.

11. Saint-Geniez M, et al. Endogenous VEGF is required for visual function: evidence for a survival role on Müller cells and photoreceptors. PLoS One. 2008;3(11):e3554.

12. Kurihara T, Westenskow PD, Bravo S, Aguilar E, Friedlander M. Targeted deletion of Vegfa in adult mice induces vision loss. J Clin Invest. 2012;122(11):4213-4217.

13. Ueno $\mathrm{S}$, et al. Prolonged blockade of VEGF family members does not cause identifiable damage to retinal neurons or vessels. J Cell Physiol. 2008;217(1):13-22.

14. Miki A, et al. Prolonged blockade of VEGF receptors does not damage retinal photoreceptors or ganglion cells. J Cell Physiol. 2010;224(1):262-272.

15. Comparison of Age-related Macular Degeneration Treatments Trials (CATT) Research Group, et al. Ranibizumab and bevacizumab for treatment of neovascular age-related macular degeneration: two-year results. Ophthalmology. 2012;119(7):1388-1398.

16. Sophie R, et al. Re: Grunwald et al.: Risk of Geographic Atrophy in the Comparison of Age-Related Macular Degeneration Treatments Trials (Ophthalmology 2014;121:150-61). Ophthalmology. 2014;121(7):e34.

17. Campochiaro PA, et al. Retinal degeneration in transgenic mice with photoreceptor-specific expression of a dominant-negative fibroblast growth factor receptor. J Neurosci. 1996;16(5):1679-1688.

18. Faktorovich EG, Steinberg RH, Yasumura D, Matthes MT, LaVail MM. Photoreceptor degeneration in inherited retinal dystrophy delayed by basic fibroblast growth factor. Nature. 1990;347(6288):83-86.

19. LaVail MM, Unoki K, Yasumura D, Matthes MT, Yancopoulos GD, Steinberg RH. Multiple growth factors, cytokines, and neu- 
rotrophins rescue photoreceptors from the damaging effects of constant light. Proc Natl Acad Sci USA. 1992;89(23):11249-11253.

20. LaVail MM, et al. Protection of mouse photoreceptors by survival factors in retinal degenerations. Invest Ophthalmol Vis Sci. 1998;39(3):592-602.

21. Dong A, Shen J, Krause M, Hackett SF, Campochiaro PA. Increased expression of glial cell line-derived neurotrophic factor protects against oxidative damage-induced retinal degeneration. J Neurochem. 2007;103(3):1041-1052.

22. Ohnaka M, et al. Long-term expression of glial cell line-derived neurotrophic factor slows, but does not stop retinal degeneration in a model of retinitis pigmentosa. J Neurochem. 2012;122(5):1047-1053.

23. McGee Sanftner LH, Abel H, Hauswirth WW, Flannery JG. Glial cell line derived neurotrophic factor delays photoreceptor degeneration in a transgenic rat model of retinitis pigmentosa. Mol Ther. 2001;4(6):622-629.

24. Byrne LC, et al. Viral-mediated RdCVF and RdCVFL expression protects cone and rod photoreceptors in retinal degeneration. J Clin Invest. 2015;125(1):105-116.

25. Chang B, Hawes NL, Hurd RE, Davisson MT, Nusinowitz S, Heckenlively JR. Retinal degeneration mutants in the mouse. Vision Res. 2002;42(4):517-525.

26. Gargini C, Terzibasi E, Mazzoni F, Strettoi E. Retinal organization in the retinal degeneration 10 (rd10) mutant mouse: a morphological and ERG study. J Comp Neurol. 2007;500(2):222-238.

27. McLaughlin ME, Sandberg MA, Berson EL, Dryja TP. Recessive mutations in the gene encoding the beta-subunit of rod phosphodiesterase in patients with retinitis pigmentosa. Nat Genet. 1993;4(2):130-134.

28. Yu DY, Cringle SJ, Su EN, Yu PK. Intraretinal oxygen levels before and after photoreceptor loss in the RCS rat. Invest Ophthal mol Vis Sci. 2000;41(12):3999-4006.

29. Braun RD, Linsenmeier RA, Goldstick TK. Oxygen consumption in the inner and outer retina of the cat. Invest Ophthalmol Vis Sci. 1995;36(3):542-554.

30. Tobe T, et al. Evolution of neovascularization in mice with overexpression of vascular endothelial growth factor in photoreceptors. Invest Ophthalmol Vis Sci. 1998;39(1):180-188.

31. Benedetti A, Comporti M, Esterbauer H. Identification of 4-hydroxynonenal as a cytotoxic product originating from the peroxidation of liver microsomal lipids. Biochim Biophys Acta. 1980;620(2):281-296.

32. Esterbauer H, Schaur RJ, Zollner H. Chemistry and biochemistry of 4-hydroxynonenal, malonaldehyde and related aldehydes. Free Radic Biol Med. 1991;11(1):81-128.

33. Shen J, et al. Oxidative damage is a potential cause of cone cell death in retinitis pigmentosa. J Cell Physiol. 2005;203(3):457-464.

34. Lee SY, et al. N-Acetylcysteine promotes long-term survival of cones in a model of retinitis pigmentosa. J Cell Physiol. 2011;226(7):1843-1849.

35. Uppu RM, Squadrito GL, Pryor WA. Acceleration of peroxynitrite oxidations by carbon dioxide. Arch Biochem Biophys. 1996;327(2):335-343.

36. Levine RL, et al. Determination of carbonyl content in oxidatively modified proteins. Meth Enzymol. 1990;186:464-478.

37. Buss H, Chan TP, Sluis KB, Domigan NM, Winterbourn CC. Protein carbonyl measurement by a sensitive ELISA method. Free Radic Biol Med. 1997;23(3):361-366.

38. Alon T, Hemo I, Itin A, Pe'er J, Stone J, Keshet E. Vascular endothelial growth factor acts as a survival factor for newly formed retinal vessels and has implications for retinopathy of prematurity. Nat Med. 1995;1(10):1024-1028.

39. Kamba T, et al. VEGF-dependent plasticity of fenestrated capillaries in the normal adult microvasculature. Am J Physiol Heart Circ Physiol. 2006;290(2):H560-H576.

40. Kamba T, McDonald DM. Mechanisms of adverse effects of anti-VEGF therapy for cancer. Br J Cancer. 2007;96(12):1788-1795.

41. Campochiaro PA, Aiello LP, Rosenfeld PJ. Anti-vascular endothelial growth factor agents in the treatment of retinal disease: from bench to bedside. Ophthalmology. 2016;123(10S):S78-S88.

42. Hombrebueno JR, Ali IH, Xu H, Chen M. Sustained intraocular VEGF neutralization results in retinal neurodegeneration in the Ins2(Akita) diabetic mouse. Sci Rep. 2015;5:18316.

43. Nguyen QD, et al. Ranibizumab for diabetic macular edema: results from 2 phase III randomized trials: RISE and RIDE. Oph thalmology. 2012;119(4):789-801.

44. Okabe K, et al. Neurons limit angiogenesis by titrating VEGF in retina. Cell. 2014;159(3):584-596.

45. Comparison of Age-related Macular Degeneration Treatments Trials (CATT) Research Group, et al. Five-year outcomes with anti-vascular endothelial growth factor treatment of neovascular age-related macular degeneration: The Comparison of AgeRelated Macular Degeneration Treatments Trials. Ophthalmology. 2016;123(8):1751-1761.

46. Peden MC, Suñer IJ, Hammer ME, Grizzard WS. Long-term outcomes in eyes receiving fixed-interval dosing of anti-vascular endothelial growth factor agents for wet age-related macular degeneration. Ophthalmology. 2015;122(4):803-808

47. Holz FG, et al. Multi-country real-life experience of anti-vascular endothelial growth factor therapy for wet age-related macular degeneration. Br J Ophthalmol. 2015;99(2):220-226.

48. Heier JS, et al. Intravitreous injection of AAV2-sFLT01 in patients with advanced neovascular age-related macular degeneration: a phase 1, open-label trial. Lancet. 2017;390(10089):50-61.

49. Komeima K, Rogers BS, Lu L, Campochiaro PA. Antioxidants reduce cone cell death in a model of retinitis pigmentosa. Proc Natl Acad Sci USA. 2006;103(30):11300-11305.

50. Komeima K, Rogers BS, Campochiaro PA. Antioxidants slow photoreceptor cell death in mouse models of retinitis pigmentosa. J Cell Physiol. 2007;213(3):809-815. 